\title{
One-Quarter-Car Active SuspensionModel Verification
}

\author{
Katerina Hyniova \\ Faculty of Information Technology, CTU in Prague, Thakurova 9, Prague 6, 16000 Czech Republic
}

\begin{abstract}
Suspension system influences both the comfort and safety of the passengers. In the paper, energy recuperation and management in automotive suspension systems with linear electric motors that are controlled by a designed $\mathrm{H} \infty$ controller to generate a variable mechanical force for a car damper is presented. Vehicle shock absorbers in which forces are generated in response to feedback signals by active elements obviously offer increased design flexibility compared to the conventional suspensions with passive elements (springs and dampers). The main advantage of the proposed solution that uses a linear AC motor is the possibility to generate desired forces acting between the unsprung (wheel) and sprung (one-quarter of the car body mass) masses of the car, providing good insulation of the car sprung mass from the road surface roughness and load disturbances. As shown in the paper, under certain circumstances linear motors as actuators enable to transform mechanical energy of the vertical car vibrations to electrical energy, accumulate it, and use it when needed. Energy flow control enables to reduce or even eliminate the demands on the external power source. In particular, the paper is focused on experiments with active shock absorber that has been taken on the designed test bed and the way we developed an appropriate input signal for the test bed that as real road disturbance acts upon the vibration absorber and the obtained results are evaluated at the end. Another important point the active suspension design should satisfy is energy supply control that is made via standard controller modification, and which allows changing amount of energy required by the system. Functionality of the designed controller modification was verified taking various experiments on the experiment stand as mentioned in the paper. .
\end{abstract}

\section{Introduction}

At the Czech Technical University in Prague various alternative strategies and innovations [2], [5] to classical passive suspension systems improving ride comfort of the passengers, providing steering stability, maximizing safety and improving handling properties of vehicles has been researched. In order to improve handling and comfort performance instead of a conventional static spring and damper system, semi-active and active suspension systems has been developed. Certainly there are numerous variations and different configurations of vibration suspension as well as control strategies [3], [4], [6]. In known experimental active systems hydraulic or pneumatic actuators usually provide the force input. As an alternative approach to active suspension system design, the research group has studied electromechanical actuators. Such actuators would provide a direct interface between electronic control and the suspension system.

In most active suspension systems, the biggest disadvantage consists in energy demands. Regarding linear electric motors, this drawback can be minimized or even eliminated because under certain circumstances there is a possibility to recuperate energy, accumulate it and use it later for the shock absorber when necessary. This way, it is possible to reduce the posted claims on the external power source as much as possible. In the next paragraphs also the proposed strategy how to control the energy distribution will be described. In order to regenerate electric power from the vibrations excited by road unevenness a new energy-regenerative active suspension for vehicles has been designed. The active system has been modeled and simulated to show the performance improvement and the performance experiments of the actuator prototype-testing stand have been carried out.

All suspension systems are designed to meet variable specific requirements. In suspension systems, mainly two most important points are supposed to be improved disturbance absorbing (videlicet passenger comfort) and attenuation of the disturbance transfer to the road (videlicet car handling). The first requirement could be understood as an attenuation of the sprung mass acceleration or as a peak minimization of the sprung mass vertical displacement. The second one is characterized as an attenuation of the force acting on the road or - in the simple car model - as an attenuation of the unsprung mass acceleration. The goal is to satisfy both the above given contradictory requirements. Satisfactory results can be achieved when an active suspension systems generating variable mechanical force acting between the sprung and unsprung masses is used. Such an actuator can be a linear electric motor. In 
comparison with traditional actuators that use revolving electro-motors and a lead screw or toothed belt, the direct drive linear motor enables contactless transfer of electrical power according to the laws of magnetic induction. The gained electromagnetic force is applied directly without the intervention of a mechanical transmission then. Linear electric motors are easily controllable and for features like low friction, high accuracy, high acceleration and velocity, high values of generated force, high reliability and long lifetime their usage as shock absorbers seems to be ideal.

\section{One-Quarter-Car Suspension Model and Shock Absorber Test Stand}

A traditional one-quarter-car model has been used to design a suspension controller design and to simulate the system behavior. The basic configuration of the model is plotted in Fig.1. The model involves unsprung (wheel) and sprung (taken as one ideal quarter of the car body mass) masses, conventional passive suspension (a spring and a damper), stiffness of the tire, and linear electric motor as actuator placed in parallel to the traditional passive suspension.

In Fig. 1:

$\mathrm{F}_{\mathrm{a}}$ control input (active suspension force) [N]

$\mathrm{m}_{\mathrm{w}}$ unsprung mass (wheel) [kg]

$\mathrm{m}_{\mathrm{b}}$ sprung mass supported by each wheel and taken as equal to a quarter of the total body mass [kg]

$\mathrm{k}_{2} \quad$ stiffness of the tire $[\mathrm{N} / \mathrm{m}]$

$\mathrm{z}_{\mathrm{r}}(\mathrm{t})$ road displacement (road disturbance) [m]

$\mathrm{Z}_{\mathrm{b}}(\mathrm{t})$ displacement of the sprung mass $[\mathrm{m}]$

$\mathrm{Z}_{\mathrm{w}}(\mathrm{t})$ displacement of the unsprung mass [m]

$\mathrm{k}_{1} \quad$ stiffness of the passive suspension [N/m]

$\mathrm{b}_{1} \quad$ damping quotient of the passive suspension $[\mathrm{Ns} / \mathrm{m}]$

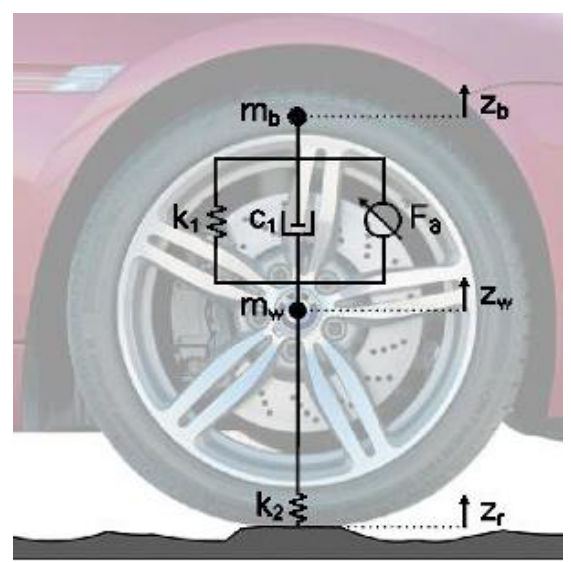

Fig.1 One-quarter-car model

The same configuration has been used for real experiments. Mechanical configuration of the test stand is obvious from Fig.2. Under the tyre there is placed another linear electric motor that uses an input experimental signal described in next paragraphs to generate road displacement (road deviations) under the running wheel.
As will be mentioned later the controller has been developed via Matlab implemented into dSpace a connected to the test stand system.

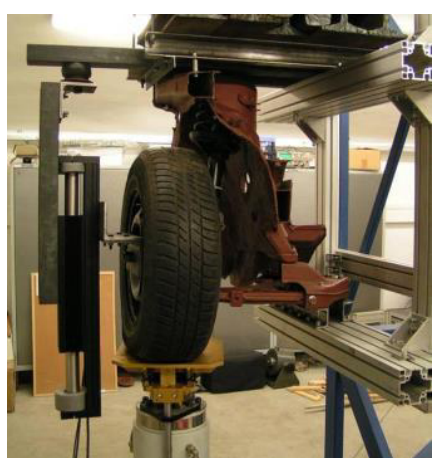

Fig2 Experiment stand

Use a two-column format, and set the spacing between the columns at $8 \mathrm{~mm}$. Do not add any page numbers.

\section{Linear Electric Motor}

Fig. 3 describes the basic principle and structure of the linear electric motor that is used as an actuator in the active suspension system. The appreciable feature of linear motors is that they directly translate electrical energy into usable linear mechanical force and motion, and vice versa. They are linear-shaped.

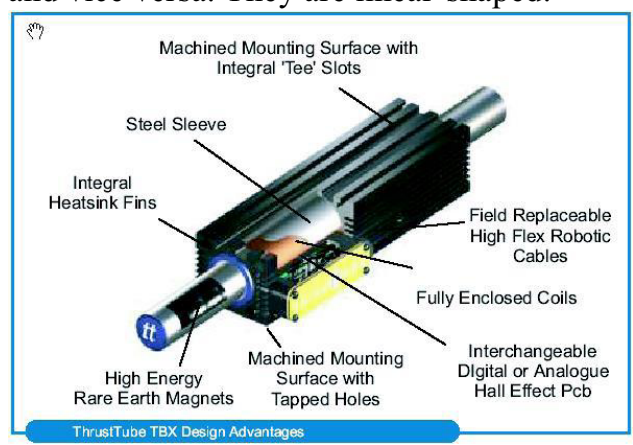

Fig3 Linear motor basic design (manufacturer spreadsheet)

Linear motor translator movements reach high velocities (up to approximately $200 \mathrm{~m} / \mathrm{min}$ ), accelerations (up to $\mathrm{g}$ multiples), and forces (up to $\mathrm{kN}$ ). The electromagnetic force can be applied directly to the payload without the intervention of a mechanical transmission. In practice, the most often used type is the synchronous three-phase linear motor.

For the suspension system design it is necessary to deal with one important question - whether it is more advantageous to include the model of the linear electric motor into the model for active suspension synthesis or if it should be used only for simulations.

Comparing advantages and disadvantages of the model inclusion, it can be said that the closed-loop provides more information so that better control results can be achieved. Unfortunately, there are also some significant disadvantages in such a situation. The first one insists in the rank of the system (and consequently the rank of the controller that increases up to 5 or more) 
and the second one is that the D matrix in the state space description of the motor model does not have full rank and that is why implementation functions are limited or too complicated. For these reasons the linear electric motor model has not been integrated in the model for active suspension synthesis.

There is another important question - whether the entire linear motor model could be omitted and a linear approximation of the desired force could be taken into account. As the mechanical and the electrical constant of the motor are inconsiderable - just about $1 \mathrm{~ms}$ each, the linear approximation is adequate and satisfactory. Moreover the linear approximation is supported by robustness of the $\mathrm{H} \infty$ control design what has been verified by various simulation results and experiments.

\section{Energy balance}

As said above, linear electric motors can recuperate energy. When the generated force is of the same direction as the suspension velocity, the energy has to be supplied into the system. Otherwise, it can be recuperated and accumulated for some future usage.

In fact, there are some non-linearity's in the recuperation process and that is why the energy management (control) is a bit difficult. Just for imagination, the 3-D plot (shown in Fig. 4) represents the force-velocity profile of the recuperated energy. It shows how much recuperated (and only recuperated) energy can be obtained under the given forces and velocities. In the plot, when the recuperated energy is equal to zero or bigger it is necessary to supply the energy into the system.

This characteristic surface gives important information regarding one of the requirements on the control system. Optimization objectives are equal to maximization of the recuperated energy (with necessary trade-offs).

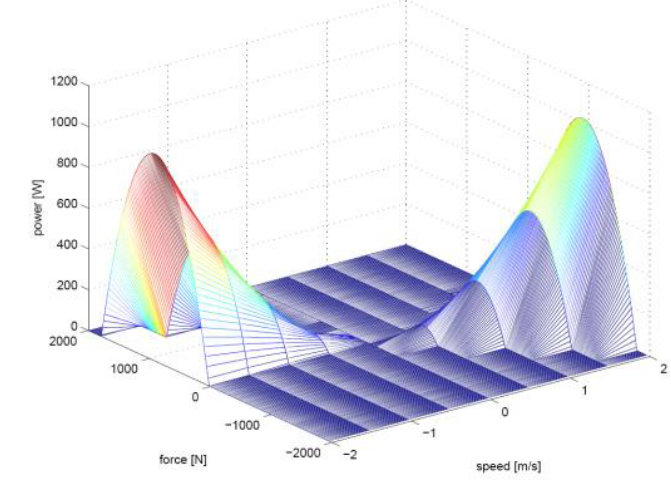

Fig.4 Recuperation aria of the linear electric motorl

\section{Experimental Signal Used to Simulate Road Displacement}

In order to bring about simulation and practical experiments at the test stand it is necessary to find a proper experimental signal that represents the road profile and excites the active suspension). Although the simplified suspension model seems to be linear the truth is that there are many nonlinear parts in the system. Now the question is what signal to generate for experimental testing to reach a true model of the uneven road under the wheel. We can define two types of input signals regarding objectives:

- to prove results of simulations and precalculations

- to test real behavior on the road

Let's start with the first objective - to verify simulation results. The best signal might be considered probably white noise because full frequency spectrum could be analyzed then. But it should be noted that the system is nonlinear and even white noise is not satisfactory. Moreover it is not possible to generate easily white noise by the test stand.

For these reasons a bump has been chosen as a signal, that often occurs on the road profiles and that can be generated by the test bed easily. This signal allows observing both directions - bump up and bump-down. Since it is not possible to generate infinite slope the following signal approximation has been used (Eq.1)(widely known approximation). Different magnitudes of the signal have been tested because the system is nonlinear. Magnitudes have been chosen according to mechanical dimensions of the suspension system:

$$
\dot{z}_{r}(t)=0.5 \pi \sin 20 \pi(t-0.1)
$$

Thus this signal is used to verify the quality of the simulation model, it does not confirm the usability of the controller on a real road surface.

More significant results can be obtained from the input signal, which is alike the road profile. A deterministic random signal is used to approximate it. The input signal for simulation is described by the following equation (2):

$$
\begin{gathered}
z_{r}=\sum_{i=1}^{n} \sqrt{\frac{\dot{\omega}_{i}}{\pi \cdot v_{x}}}\left\{\operatorname{Re}\left(\frac{b_{o}}{-\omega_{i}^{2}+a_{1} j \omega_{i}+a_{o}}\right) \cdot \cos \left(\omega_{i} t+\alpha\right)+\right. \\
\left.+\operatorname{Im}\left(\frac{b_{o}}{-\omega_{i}^{2}+a_{1} j \omega_{i}+a_{o}}\right) \cdot \sin \left(\omega_{i} t+\alpha\right)\right\} \\
\mathrm{b}_{\mathrm{o}}=0.121 \cdot \mathrm{v}_{\mathrm{x}} \\
\mathrm{a}_{\mathrm{o}}=2.249 \cdot \mathrm{v}_{\mathrm{x}} \\
\mathrm{a}_{1}=30.36 \cdot \mathrm{v}_{\mathrm{x}}
\end{gathered}
$$

where $\mathrm{v}_{\mathrm{x}}$ represents the car velocity.

Thus resulted signal is obtained as a superposition of the sinusoids with deterministic "random" angles $(\alpha$ in Eq.2). In this case 128 random angles have been calculated. The used pseudo-random signal is plotted in Fig.5. 


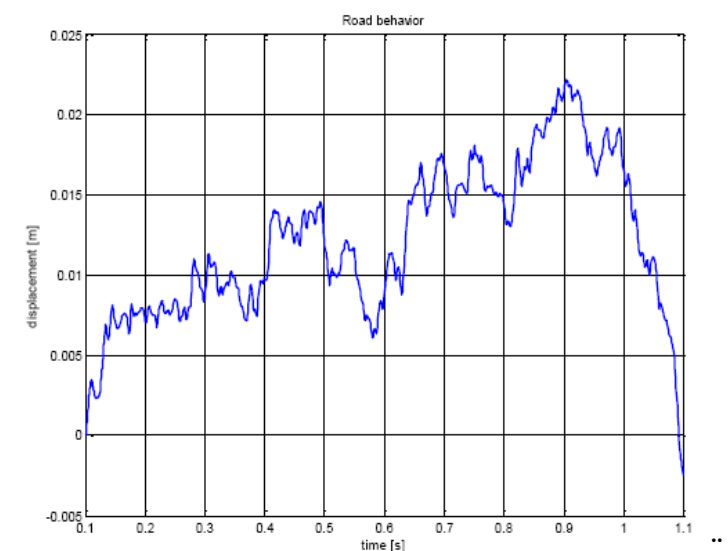

Fig5 Random signal approximation

\section{On The Control loop}

A robust controller is necessary to design for the suspension system, because the system parameters often vary in a wide range. Especially the body mass varies for every single drive. For this reason $\mathrm{H} \infty$ control theory [3] has been chosen for controller design [2] as the ideal controller design. Matlab Toolbox procedures have been used for $\mathrm{H} \infty$ controller computation.

Nevertheless the standard $\mathrm{H} \infty$ controller cannot handle energy consumption. Modification of the controller had to be done during experiments. Additional input, which controls energy demands was supposed to be connected to the controller. Master controller then can use this input to keep energy balance (Design of the master controller is not involved in the paper.). The structure of the modified controller is shown in Fig.6.

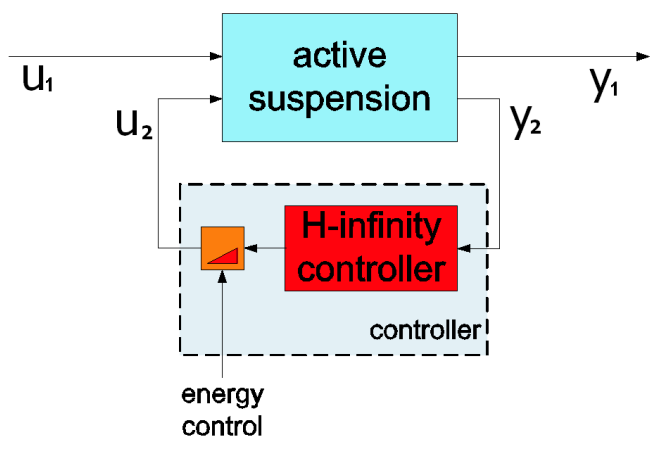

Fig6 Modified controller structure

Than energy management is supposed to be controlled by an external signal (Fig.6) depending on the car and road parameters, i.e. on the energy accumulator capacity and the road profile, respectively.

First possible way to control energy fed into the suspension system consist in the analysis of the driving conditions and cyclic re-computing of the control signal in real time. For high sampling frequency (over $1 \mathrm{kHz}$ ) and because of the performance of the controller can not be guaranteed for all operating conditions this approach has been rejected.

The second possibility is to control the energy consumption by controller deterioration. Then the designed controller is reliably robust and the active suspension system is relatively stable. Simply said, the controller deterioration sort of devalues the suspension performance, but enables to gain some energy to be stored. Strictly speaking, let us assume two kinds of driving conditions:

- the terrain /road surface the car is going on is very rough and uneven and there is enough energy stored in the accumulator system- then the controller works in the standard mode, the linear motor consumes energy from the accumulator (supercapacitors) and the suspension performance is preserved.

- the terrain /surface under the car wheels is relatively smooth and there is not enough energy stored in the accumulator system (supercaps) as it was consumed because of the situation described above. The external signal provides the information to the controller to deteriorate its performance and to reduce the energy consumption. The deterioration is stated by the desired force attenuation so that the suspension gets devaluated.

If the force is completely attenuated the suspension system works only via the passive suspension part while the linear electric motor works as a generator generating electrical energy to be stored in the accumulator system. Of course, the suspension performance is devaluated now (to the passive suspension level in the worst case).

That is the basic description how the control loop with the modified controller works. Now, let us show some results (percentage indicators) of the real experiments taken at the test stand that endorse presumed above.

\section{Results}

Deterministic random signal stated in (2) has been used for real experiments taken on the shock absorber test stand. Two important things should be followed during experiments - suspension comfort improvement and energy consumption (see Fig. 7). In Fig 7, the road profile input, energy consumption, and corresponding body displacement for standard controller setting are displayed.

As an indicator of the comfort improvement body (sprung) mass displacement can be taken.

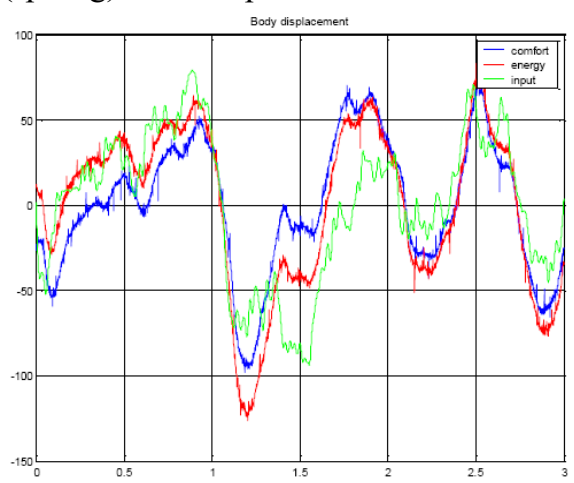

Fig7 Sprung mass displacement

Fig. 8 shows two curves - energy demand for standard energy consumption setting (called "comfort setting") and energy demands for lower consumption (called "energy setting").Both curves were measured excited 
with the same input- the deterministic random signal (road profile). Actually, negative values of energy in the figure represent the recuperated energy.

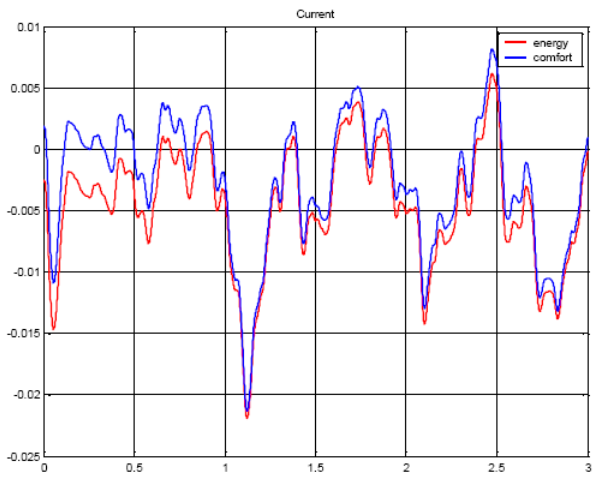

Fig8 Energy demands (demands on electrical current) $\quad[1] K$.

Let's show the results as mean values of "comfort" and "energy" indicators stated in [1]. Tab.1 involves mean values for the body displacement as absolute values of the defined body displacement indicator and also as a percentage of its improvement.

Table 1 Displacement mean values

\begin{tabular}{|l|c|c|}
\hline & Mean value & Percentage \\
\hline $\begin{array}{l}\text { Body displacement }- \\
\text { comfort }\end{array}$ & 38.6 & $100 \%$ \\
\hline $\begin{array}{l}\text { Body displacement }- \\
\text { energy }\end{array}$ & 47.4 & $123 \%$ \\
\hline
\end{tabular}

The first line shows that for standard controller setting the comfort indicator (38.6) is taken as $100 \%$. In the second line, when the controller was deteriorated, the comfort is devaluated up to 47.4 , i.e. to $123 \%$.

Tab.2 involves mean values of energy indicator. Lower value corresponds to the lower energy demand. Similarly the first line shows that for standard controller setting the comfort indicator (2.689) is taken as $100 \%$. In the second line, when the controller was deteriorated, the energy indicator is devaluated up to 1.598 , i.e. to $59 \%$ then.

Briefly, controller deterioration causes comfort devaluation to $123 \%$ while energy consumption decreases to $59 \%$.

Table 2 Power mean values

\begin{tabular}{|l|c|c|}
\hline & Mean value & Percentage \\
\hline Comfort setting & 2.689 & $100 \%$ \\
\hline Energy setting & 1.598 & $59 \%$ \\
\hline
\end{tabular}

\section{Conclusion}

In this paper the $\mathrm{H} \infty$ controller for active suspension with linear electric motor has been used for experiment done on the experiment stand. An experiment signal for real road profile simulation has been developed and then it has been used for experiments. The method for the direct real-time energy control with respect to reduction of the energy consumption has been used. Experiments verified results of the simulations and showed that it is possible to change energy demands according to the road profile and status of the energy storage in the car (battery or super-capacitor). The method can be extended to general plants with considerable energy demands, where the decreasing actuator signal in a given range can preserve the system stability. Thus this controller with linear motor as an actuator can be used in any suspension system.

This research has been supported by the MSMT project No. 1M6840770002 "Josef Bozek's Research Center of Combustion Engines and Automobiles II" and MSMT project INGO No. LG14005.

\section{References}

$1 \quad K$ Hyniova and J. Honcu: Active Suspension System -- Experiments, in Proceedings of the 4th WSEAS/IASME International Conference on DYNAMICAL SYSTEMS and CONTROL, Corfu, Greece, October 26- 28, 2008

2 A. Kruczek and A. Stribrsky, A: Full-car Model for Active Suspension - Some Practical Aspects, in Proccedings of IEEE International Conference on Mechatronics, Istanbul, Turkey, 2004

3 Nastac, S.: Simulation of active Vibration Isolation with Fuzzy Logic Device, In: Proceedings of the $10^{\text {th }}$ international Conference on mathematical and computational methods in Science and Engineering (MACMESE'08), pp. 346-351) Brasov, 2008.

4 ] G.C.D. Sousa and B.K. Bose, A fuzzy set theory based control of a phase-controlled converter DC machine drive, IEEE Trans. Industrial Applications 30 (1994)

5 ] Stribrsky, A. - Hyniova, K. - Honcu, J.: Reduction of Vibrations in Mechanical Systems, Proceedings of Workshop 2001, Part A, pp.174-175, CTU Prague, 2001

6 Zhou and J. C. Doyle: Essentials of Robust Control, Prentice Hall, 1998 